\title{
Correction to: A new atherinomorine genus Doboatherina (Atheriniformes: Atherinidae) with a review of included species
}

\author{
Daichi Sasaki $^{1,2} \cdot$ Seishi Kimura ${ }^{1,3}$
}

Published online: 9 January 2020

(c) The Ichthyological Society of Japan 2020

\section{Correction to: Ichthyological Research https://doi.org/10.1007/s10228-019-00718-5}

The original publication of this article contains erroneous description and figure, which are corrected as below.

In "Comparison" of Doboatherina aetholepis (Kimura, Iwatsuki and Yoshino 2002), the second sentence reads,

"Although D. aetholepis shares a long spatular outgrowth posteriorly on the predorsal scales ..... (upper jaw length $32-41 \%$ SL vs. 29-34\%)". However, the proportion of upper jaw length should be a ratio to head length (HL), not standard length (SL).

Figure 8 is incorrect. The correct figure is as follows.

Publisher's Note Springer Nature remains neutral with regard to jurisdictional claims in published maps and institutional affiliations.

The original article can be found online at https://doi.org/10.1007/ s10228-019-00718-5.

Seishi Kimura

kimura-s@bio.mie-u.ac.jp

1 Fisheries Research Laboratory, Mie University, 4190-172

Wagu, Shima-cho, Shima, Mie 517-0703, Japan

2 Present Address: Mie Prefecture Fisheries Research Institute, 3564-3 Hamajima, Hamajima-cho, Shima, Mie 517-0404, Japan

3 Present Address: Graduate School of Bioresources, Mie University, 1577 Kurimamachiya-cho, Tsu, Mie 514-8507, Japan 


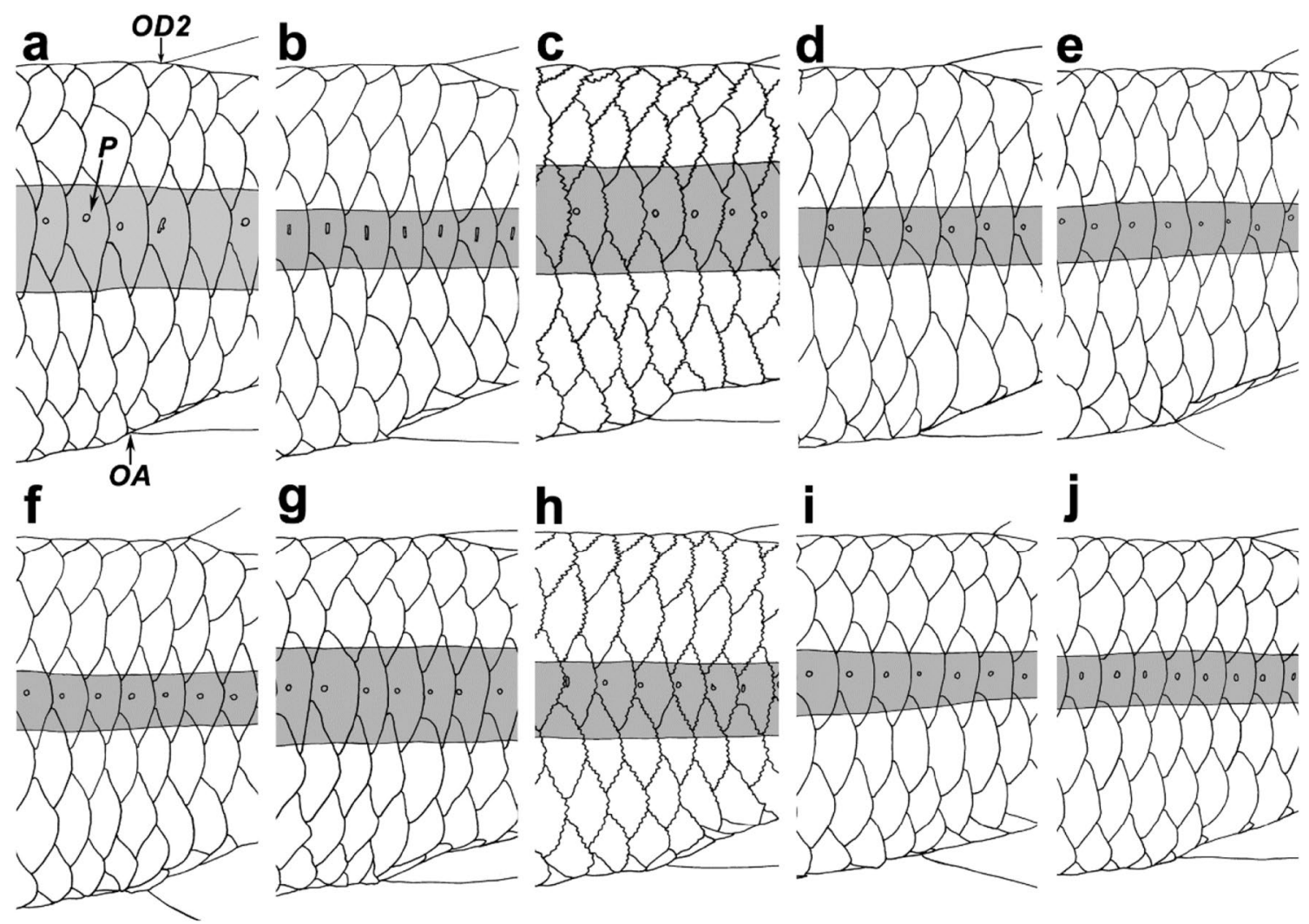

Fig. 8 Midlateral band and lateral scale laws at the level of second dorsal- and anal-fin origins in Doboatherina aetholepis (a), D. balabacensis (b), D. bleekeri (c), D. duodecimalis (d), D. iwatsukii sp. nov. (e), D. magnidentata sp. nov. (f), D. salangensis sp. nov. (g), D. valenciennei (h), D. woodwardi (i), D. yoshinoi sp. nov. (j). a FRLM 14644, paratype, $69 \mathrm{~mm}$ SL, Ambon, Indonesia; b USNM 136802, neotype, $64 \mathrm{~mm}$ SL, Ticao I., Philippines; c FRLM 36718, 66 mm SL, Owase, Mie, Japan; d FRLM 36073, 73 mm SL, Ambon,
Indonesia; e MUFS 14670, paratype, $44 \mathrm{~mm}$ SL, Phuket, Thailand; f KAUM-I. 24170, paratype, $64 \mathrm{~mm} \mathrm{SL}$, Bang Saen, Chonburi, Thailand; g FRLM 47561, paratype, $48 \mathrm{~mm}$ SL, Phuket, Thailand; h FRLM 35363, 70 mm SL, Songkhla, Thailand; i URM-P 17407, 55 mm SL, Uruma, Okinawa, Japan; j FRLM 39132, paratype, $60 \mathrm{~mm}$ SL, Iriomote I., Okinawa, Japan. OD2, second dorsal-fin origin; $O A$, anal-fin origin; $P$, central pit of midlateral scales 